\title{
Mate preference: a possible causal mechanism for a moving hybrid zone
}

\author{
C. L. BRONSON*, THOMAS C. GRUBB, JR*, GENE D. SATTLER† \& MICHAEL J. BRAUN \\ *Behavioral Ecology Group, Department of Evolution, Ecology, and Organismal Biology, The Ohio State University \\ $\dagger$ Department of Biology and Chemistry, Liberty University \\ \$Analytical Biology and Department of Systematic Biology, National Museum of Natural History, Smithsonian \\ Institution
}

(Received 9 July 2001; initial acceptance 3 December 2001; final acceptance 2 July 2002; MS. number: A9111)

\begin{abstract}
The study of patterns and underlying mechanisms within hybrid zones may provide insight into speciation. Black-capped (Poecile atricapillus; BC) and Carolina (P. carolinensis; CA) chickadees hybridize in an east-west band in the U.S.A. from New Jersey to Kansas. Within the past century, the Ohio portion of this hybrid zone and the CA range to the south have been moving northward while the $\mathrm{BC}$ range has retracted. We examined mate preference in females of both species as one possible causal mechanism for this shift. To be conservative about the nomenclature and results, the samples are referred to as either 'BC-like' or 'CA-like' due to the observed genetic introgression in the study individuals. Given a choice within an aviary setting, in the aggregate, BC-like and CA-like females that had not observed the direct social interactions between a dyad of a BC-like male and a CA-like male preferred to associate with the BC-like male. In nature, both species form within-sex dominance hierarchies. In the aviary, CA-like males dominated BC-like males unless a CA-like male was substantially smaller. Once females of both types had observed the physical interaction of a particular heterospecific dyad, they associated preferentially with the dominant male, regardless of species type. Thus, the effect of CA male intrasexual dominance on female mate preference may be contributing to the northward movement of the hybrid zone.
\end{abstract}

(c) 2003 Published by Elsevier Science Ltd on behalf of The Association for the Study of Animal Behaviour.

A focal area in the study of organisms is the nature of genetic cohesion within groups. Hybrid zone dynamics are a fertile area for such research because of the exchange of genes between such distinct groups (Hewitt 1988; Harrison 1990, 1993). In a variety of taxa, all or portions of hybrid zones have been observed to move over time (e.g. Arntzen \& Wallis 1991; Hairston et al. 1992; Shaw et al. 1993; Rohwer \& Wood 1998; Shapiro 1998). For many hybrid zones, each subsection of a zone has individualized ecological constraints and evolutionary history that greatly impact the observed dynamics in that area (e.g. Bert \& Harrison 1988; Hairston et al. 1992; Kallioinen

Correspondence and present address: C. L. Bronson, The Ohio State University, Department of Internal Medicine, Divison of Immunology, 425 Heart and Lung Research Institute, 473 West 12th Avenue, Columbus, OH 43210-1293, U.S.A. (email: bronson.5@osu.edu).T. C. Grubb, Jr, The Ohio State University, Department of Evolution, Ecology and Organismal Biology, 1735 Neil Avenue, Columbus $\mathrm{OH}$ 43210-1293, U.S.A. G. D. Sattler is at Liberty University, Department of Biology and Chemistry, 1971 University Boulevard, Lynchburg, VA 24502, U.S.A. M. J. Braun is at the Analytical Biology and Department of Systematic Biology, Smithsonian Institution, 4210 Silver Hill Road, Suitland, MD 20746, U.S.A. et al. 1995). Correlational evidence indicates that movement in a warbler hybrid zone might result from male aggression and mate choice (Pearson 2000; Pearson \& Rohwer 2000), rather than from either naturally occurring habitat transition or anthropogenic modification of the habitat (Pearson \& Manuwal 2000). Similarly, experimental evidence of male territorial behaviour linked with anthropogenic modification of the habitat might explain movement of a magpie hybrid zone (Kallioinen et al. 1995).

Black-capped (Poecile atricapillus; BC) and Carolina (P. carolinensis; $\mathrm{CA})$ chickadees are known to hybridize in many areas along the common distribution border (e.g. Missouri: Braun \& Robbins 1986; Sawaya 1990; Ohio: Grubb et al. 1994; Virginia and West Virginia: Sattler 1996; Sattler \& Braun 2000; Pennsylvania: R. L. Curry, personal communication). While quite similar in appearance, the two species do have distinguishable phenotypes (Pyle 1997).

In Ohio, the $\mathrm{BC}$ distribution abuts the north edge of the CA distribution. In the 1880 s, CAs were described as permanent residents within the southern portion of the state (Wheaton 1882). Within Ohio in the early 1940s, 
the east-west oriented hybrid zone was probably located across the middle of the state approximately along the current location of U.S. Interstate 70 (Trautman 1940). Currently, while still in an east-west orientation, the zone is located about $100 \mathrm{~km}$ farther north in the state, approximately along U.S. Highway 30 (Grubb et al. 1994; Peterjohn 2001). In other words, the BC distribution has been receding northward.

Two potential factors that may influence the movement of this portion of the chickadee hybrid zone are mate choice and social dominance. Mate choice in a mixed-species context has not been addressed previously in BC and CA. However, studies of mate choice in individual chickadee species have yielded results germane to interspecific mate choice. Both species form linear, within-sex dominance hierarchies in the winter (Hartzler 1970). Within such winter flocks, females have been shown to desert their mate in favour of a mate with a higher rank (Smith 1991; Otter \& Ratcliffe 1996). Potential benefits a female could gain from making such a switch are increased winter survival (Ekman 1990; Hogstad 1992; Lemmon et al. 1997) and a larger breeding territory (Smith 1976). Because male dominance appears to be an important causal factor in female mate choice in chickadees, we investigated: (1) whether BC and CA females prefer a conspecific or heterospecific male, (2) whether such females prefer a dominant or subordinate male, and (3) the relative strengths of male species identity and male dominance status as determinants of female preference for a male.

\section{METHODS}

\section{Subjects}

During January-March 1998 and 1999, chickadees were captured as pairs using either mist nets or remotecontrolled feeder traps (Pierce \& Grubb 1979). All birds were subsequently genotyped for five diagnostic marker loci (see below), and the large majority of subjects possessed either mostly BC or CA alleles. However, because of the presence of apparent hybrids in the study (see Results), the terms BC-like and CA-like are used for the samples in order to be conservative about species nomenclature. The 10 BC-like pairs were taken from Seneca County, Ohio $\left(41^{\circ} 4^{\prime} \mathrm{N}, 82^{\circ} 53^{\prime} \mathrm{W}\right.$, approximately $25 \mathrm{~km}$ north of the centre of the hybrid zone). The $10 \mathrm{CA}$-like pairs came from Morrow $\left(40^{\circ} 24^{\prime} \mathrm{N}, 82^{\circ} 49^{\prime} \mathrm{W}\right)$ and Union $\left(40^{\circ} 14^{\prime} \mathrm{N}, 83^{\circ} 22^{\prime} \mathrm{W}\right)$ Counties, Ohio; both areas are approximately $45 \mathrm{~km}$ south of the centre of the hybrid zone.

At the time of capture, each bird was banded with a U.S. Fish and Wildlife Service aluminium band for identification. The use of colour bands for individual identification has been shown to influence female mate choice in several avian species (Burley 1986, 1988; Metz \& Weatherhead 1991; Johnson et al. 1993). Fortunately, such a potential complication from using colour bands was avoidable in this experiment. First, each heterospecific dyad of males employed in the experiment was always in physical isolation from the experimental female in the aviary. Second, within each male dyad, the BC-like male had readily apparent whiter wings (secondary coverts and feather margins) and a longer tail; therefore, the males were individually identifiable when housed together.

Each bird was weighed at capture to the nearest $0.1 \mathrm{~g}$ using a spring balance. Unflattened wing chord and tail length were measured to the nearest $0.5 \mathrm{~mm}$, and tarsometatarsus length to the nearest $0.1 \mathrm{~mm}$. Sex was determined based on behavioural observation prior to capture (i.e. males dominant to females) and the relative size of the pair (Desrochers 1990; Smith 1991). We could not accurately determine previous reproductive activity, previous mating status, previous mating partner or age for the experimental individuals.

Each bird was scored on four morphological characters, whiteness of secondary wing coverts, whiteness of secondary feather margins, whiteness of tail margins and straightness of the lower margin of the 'bib'. The first three were scored similarly: 0 if white (BC-like), 1 if tan, 2 if grey (CA-like). The last was scored a 0 if jagged (BC-like) and 1 if straight (CA-like). As a percentage of the possible total of seven, pure $\mathrm{BC}$ and CA phenotypes would yield $0 \%$ and $100 \%$, respectively.

For genetic analysis, a 50-75- $\mu$ l blood sample from each bird was taken in a heparinized capillary tube from a brachial vein punctured with a 25-gauge needle. Each blood sample was placed in $1 \mathrm{ml}$ of lysis buffer $(100 \mathrm{mM}$ Tris at pH 8.0, $100 \mathrm{mM}$ EDTA, $10 \mathrm{mM} \mathrm{NaCl}, 0.5 \%$ SDS; Longmire et al. 1988) and stored at $4^{\circ} \mathrm{C}$ for later DNA extraction.

All birds were maintained on ad libitum black oil sunflower seeds, a daily mealworm supplementation and ad libitum water/snow in individual welded-wire cages at the aviary site $\left(40^{\circ} 24^{\prime} \mathrm{N}, 82^{\circ} 49^{\prime} \mathrm{W}\right)$. To limit the females' experience with stimulus males, the two sexes were housed in different buildings. Within each building, the birds were visually but not acoustically isolated from each other. Both buildings were maintained at ambient temperature and photoperiod.

\section{Genetic Analysis}

DNA was extracted from the blood samples at The Ohio State University in the laboratory of Patricia G. Parker. Each blood sample had $200 \mu \mathrm{g}$ of Proteinase K added and was then incubated at $55^{\circ} \mathrm{C}$ overnight. Five extractions were performed, two with phenol, two with 25:24:1 phenol:chloroform:isoamyl alcohol, and one with $24: 1$ chloroform:isoamyl alcohol. Then, the remaining aqueous phase of each sample was dialyzed extensively against $\mathrm{TNE}_{2}(10 \mathrm{mM}$ Tris at $\mathrm{pH} 7.4,10 \mathrm{mM} \mathrm{NaCl}, 2 \mathrm{mM}$ EDTA). The extracted DNA samples were stored at $4^{\circ} \mathrm{C}$ for later genetic analysis.

The concentration and purity of each extracted DNA sample were assessed using spectrophotometric measures of optical densities at 260 and $280 \mathrm{~nm}$. The concentration estimate and integrity (sheared versus unsheared) of each sample were assessed by running (at $80 \mathrm{~V}$ ) $1.0 \mu \mathrm{g}$ of undigested DNA through a $0.8 \%$ agarose gel, staining the resulting gel with ethidium bromide and viewing it on a 
UV light box. All the samples were determined to be unsheared.

Genetic analyses were performed at the Smithsonian Institution's Laboratory of Molecular Systematics following the methods of Sattler \& Braun (2000). Five probes were used to identify restriction fragment length patterns diagnostic for CA and BC on Southern blots (Sambrook et al. 1989). First, EcoR I digests were probed with a 1200-bp fragment of the domestic chicken, Gallus gallus domesticus, oncogene ski ( $\mathrm{Li}$ et al. 1986; Sawaya 1990; Sattler \& Braun 2000). Second, Bgl II digests were probed with a randomly cloned fragment of CA DNA designated RP104 (Sattler 1996). Third, Ava II digests were probed with a randomly cloned fragment of CA DNA designated RP7 (Sattler 1996). Fourth, Pst I digests were probed with a randomly cloned 4000-bp fragment of tufted titmouse, Baeolophus bicolor, DNA designated C7 (Sawaya 1990; Sattler \& Braun 2000). Fifth, Pst I digests were also probed with house finch, Carpodacus mexicanus, mitochondrial DNA (mtDNA) (Mack et al. 1986; Sawaya 1990; Sattler \& Braun 2000) purified by subcellular fractionation and $\mathrm{CsCl}$ equilibrium-gradient centrifugation (Dowling et al. 1990). The five resulting genotypes were combined into an index calculated as the percentage of CA restriction fragment length alleles present in an individual. The combination of Pst I/mtDNA has only one variant because mtDNA is haploid. The combination of Pst I/C7 has only one allele in the females because it is a sex-linked marker and females are the heterogametic sex in birds. Thus for males, the genetic index was based on nine marker alleles, one for the combination of Pst I/mtDNA and two for each of the other enzyme and nuclear probe combinations. For females, the genetic index was based on eight alleles, one each for Pst I/mtDNA and Pst I/C7 and two for each of the other enzyme and probe combinations.

\section{Aviary}

The aviary building was situated in a meadow and more than $20 \mathrm{~m}$ from wooded areas. The animal use portion of the aviary building (response and stimulus sections in Fig. 1) had a sand floor, welded-wire and translucent corrugated fibreglass walls, and a welded-wire ceiling open to the sky. The observation chamber had a wooden floor, opaque corrugated fibreglass walls, an insulated ceiling, and was separated from the response section by a 'one-way' Plexiglas wall. The animal use portion of the aviary was permanently divided by welded wire into two main areas, the response section and the stimulus section. Within the response section, a floor-to-ceiling wall was present at the midline of the aviary extending $1.47 \mathrm{~m}$ from the section divider. This wall limited the number of perching locations in the response section from which both stimulus subsections were visible. A permanent wall $1.04 \mathrm{~m}$ high extended along the full length of the stimulus section midline, while a series of translucent corrugated fibreglass panels were suspended from the ceiling above the permanent wall. The combination of this permanent wall and the removable fibreglass panels above it divided the stimulus section into two visually

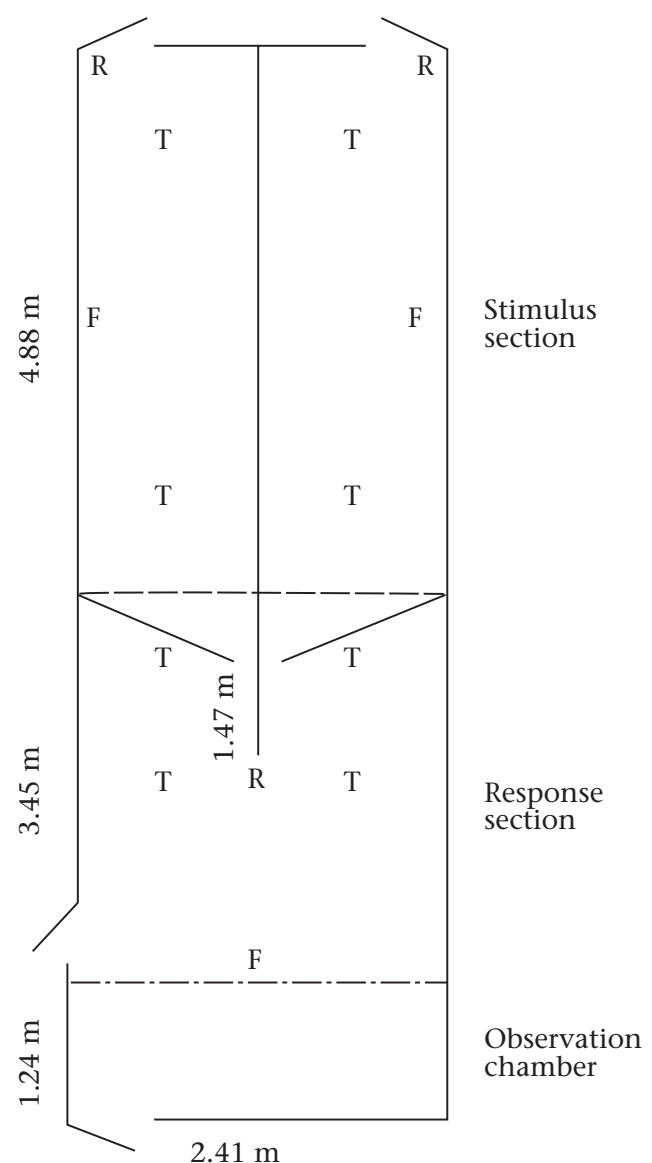

Figure 1. An overhead diagram of the aviary building. Ceiling height $=2.05 \mathrm{~m}$, height of the permanent portion of stimulus section divider $=1.04 \mathrm{~m}$. $\mathrm{T}$ =artificial tree; $\mathrm{F}=$ remote-controlled feeder trap; $\mathrm{R}=$ roosting chamber. Solid lines are corrugated fibreglass, the dashed line is welded-wire, and the dash-dot line is one-way Plexiglas.

isolated subsections of equal size. The series of corrugated fibreglass panels could be dropped down into the permanent wall to allow a bird to fly throughout the entire stimulus section. Translucent corrugated fibreglass doors were used to temporarily allow either visual access (doors open) or no visual access (doors closed) between the response section and stimulus subsections.

The response section and two stimulus subsections each contained artificial trees, a roosting chamber and a remote-controlled feeder trap (Fig. 1). Two of the response section's four artificial trees had perches allowing visual access only to the left stimulus subsection. Likewise, two artificial trees in this section had perches with potential visual access only to the right stimulus subsection. Each stimulus subsection had two artificial trees. Each artificial tree consisted of a piece of wood $(3 \times 3 \times 185 \mathrm{~cm})$ suspended from the welded-wire ceiling by a hook screwed into one end. Each artificial tree contained 12 dowel-rod perches $(22 \times 1 \mathrm{~cm})$, three on each face.

Each of the three roosting chambers had a sawdust floor and was fashioned from an artificial nesting snag (Grubb \& Bronson 1995; Fig. 1). In the response section, 
Table 1. Daily components of the mate preference trials of female chickadees

\begin{tabular}{|lcccc|}
\hline Component & Day & $\begin{array}{c}\text { Dyad of males } \\
\text { present in } \\
\text { stimulus subsections }\end{array}$ & $\begin{array}{c}\text { Female could } \\
\text { view the } \\
\text { stimulus subsections }\end{array}$ & $\begin{array}{c}\text { Female had observed the } \\
\text { dominance relationship of } \\
\text { the dyad of males }\end{array}$ \\
\hline Control 1 & 1 & No & No & No \\
Control 2 & 2 & No & Yes & No \\
Control 3 & 3 & Yes & No & No \\
Treatment 1 & 4 & Yes & Yes & Yes \\
Treatment 2 & 5 & Yes & Yes & \\
\hline
\end{tabular}

Translucent fibreglass doors were opened or closed to control visual access between the response section and the stimulus subsections.

the roosting chamber was attached to the midline wall near the ceiling. The opening of the roosting chamber was oriented towards the observation chamber. In each stimulus subsection, the roosting chamber was positioned farthest from the response section in the corner of the two outside walls. The openings of these roosting chambers were oriented towards the response section and observation chamber. The remote-controlled feeder trap in the response section was attached midway along the wall between the response section and the observation chamber with its opening oriented towards the response section. The remote-controlled feeder trap in each of the two stimulus subsections was attached midway along the outside wall with its opening oriented towards the wall subdividing the stimulus section.

While in the aviary, birds received ad libitum black oil sunflower seeds, daily mealworm supplementation, and ad libitum water/snow. The aviary's sections were not acoustically isolated either from each other or the surrounding habitat.

\section{Experimental Procedure}

The ideal design for a mate preference experiment would expose each female to an unique heterospecific dyad of males. However, such a design would require twice as many males as females. The availability of birds and the use of these birds in a subsequent experiment requiring male-female pairs constrained this study to use equal numbers of males and females. Therefore, each dyad of males consisting of one BC-like male and one CA-like male was used as the stimulus for one BC-like female and one CA-like female. During the course of the presentation of a dyad of males to the first female, we allowed the males to physically interact and establish a dominance hierarchy. Therefore, when the dyad of males was presented to the second female, they could potentially have conveyed their dominance status to her (e.g. via vocalizations, pheromones) even when the dyad of males were both physically and visually isolated from each other. To limit any bias from this potential information transfer, we presented each dyad of males in random order to the two females. No female was presented with a dyad of males containing a conspecific male captured at the same location where she was captured.
Five days (plus an initial day of acclimation) were required to collect data for each female (Table 1). Ten females of each species were used. A data point consisted of the female's presence on the side of the response section near one side of the stimulus section. The midline location of the wall in the response section was used for determining a female's position. The location of the artificial trees for perching and the Plexiglas wall (no perching opportunity) separating the observation chamber and response section minimized the number of entries into a 'neither' category. The 'neither' category consisted of midline activities (perching on the feeder or roosting chamber, or feeding at the feeder). A data collection session consisted of 30 data points collected $30 \mathrm{~s}$ apart (instantaneous sampling; Lehner 1979) beginning approximately $90 \mathrm{~min}$ after sunrise and at least $30 \mathrm{~min}$ after the observer had entered the observation chamber.

The female was not provided with a refuge area where she could go and not participate in the experiment. As a result, it is possible that some of her time spent in association with a male should not be attributed to her attempting to associate with that male. The area of the aviary is not much larger than the recommended distance for calculating association indices in nature (Smith 1991). Unless the female systematically changed the area she preferred in accordance with the treatments, both the categorization of a location preference based on statistical significance (versus a majority) and the use of numerous controls should have limited likelihood that females were not associating with particular males. Additionally, due to their identical nature with the data presented here, the results of two later 15-min sessions (separated by $45 \mathrm{~min}$ ) each day per female were not included. Thus, it is unlikely that a female might have systematically changed her area preference with treatment (even across three observational periods) based on a pattern of 'loafing' rather than associating with a male.

The afternoon before the first day of data collection, we released a female into the response section with the doors to the stimulus subsections closed. This procedure allowed the female to acclimate to the response section of the aviary without visual access to the stimulus subsections and without a dyad of males present. The first day of data collection, Control 1, served two purposes. First, it was used to determine whether a female had a positional bias in the response section when she was without visual 
access to the stimulus subsections and without a dyad of males present. Second, it was used as a within-female control (see Data Analysis) for a female's responses to a dyad of males in the stimulus subsections when she could not see them.

After Control 1 had been completed, we opened the doors to the stimulus sections so the female would acclimate to the response section of the aviary with visual access to the stimulus subsections without a dyad of males present. The second day of data collection, Control 2 , served three purposes. First, it was used to determine whether a female had a positional bias in the response section when she had visual access to the stimulus subsections without a dyad of males present. Second, it was used as a within-female control (see Data Analysis) for when she could see a dyad of males in the stimulus subsections but had not yet observed the dyad physically interact (i.e. dominance unobserved). Third, it was used as a within-female control for when she could see a dyad of males after having observed them physically interact (i.e. dominance observed).

After Control 2 had been completed, we closed the doors to the stimulus subsections and released (within seconds) each member of a dyad of males into a different, randomly determined, stimulus subsection. We allowed the female to acclimate to the response section of the aviary without visual access to the stimulus subsections and with a dyad of males present. Also, we allowed each male of the dyad to acclimate to his stimulus subsection without visual access to the response section or to the other stimulus subsection. The third day of data collection, Control 3, determined whether a female had a preference for one of the two males of a dyad based on nonvisual characteristics. This control was included because a variety of factors (i.e. vocalizations, pheromones) could not be eliminated from the experimental design without greatly altering the aviary (i.e. anechoic sections, independent air systems for each section) and/or the males (i.e. surgically preventing vocalizations).

After Control 3 had been completed, we opened the doors to the stimulus subsections, allowing the female to acclimate to the response section of the aviary with visual access to the stimulus subsections and the dyad of males present. Also, each male of the dyad could acclimate to its stimulus subsection with visual access to the response section and the female, but without visual access to the adjoining stimulus subsection or the male within it. The fourth day of data collection, Treatment 1 , served two purposes. First, it was used to determine whether a female had a preference for one of the two males of a dyad without having observed the males physically interact. Second, it was used in determining whether a female switched her preference after observing the dominance relationship between the two males of a dyad.

After Treatment 1 had been completed, we lowered the fibreglass panels suspended between the two stimulus subsections into the permanent, dividing wall. Because the two males had precedence on one half of the aviary and were similarly new to the respective other half, the seniority effect (Glase 1973; Hogstad 1987) should have been minimized. From the observation chamber, we monitored continuously the dyad of males until their dominance relationship had been determined. One male was scored as dominant each time he chased the other one, supplanted it from a perch, or caused it to wait before entering a feeder. When 10 consecutive interactions favoured one male, he was considered the dominant of the dyad, a decision typically reached within $30 \mathrm{~min}$. However, the dyad of males was still allowed to interact for $2 \mathrm{~h}$, all the while in view of the female in the response section. Since each dyad of males was presented to two females, we collected dominance records twice for each dyad. Male dominance was used as a factor in several analyses (see Data Analysis).

At the completion of their interaction, the two males, without being handled, were confined into their original subsections and the dividing fibreglass panels were suspended from the ceiling. We allowed the female to acclimate to the response section of the aviary with visual access to the stimulus subsections and with a dyad of males present whose dominance relationship she had had a chance to observe. Also, we allowed each male of the dyad to acclimate to his stimulus subsection with visual access to the response section and the female, but without visual access to the other stimulus subsection. The fifth day of data collection, Treatment 2, served two purposes. First, it was used to determine whether a female had a preference for one of the two males of a dyad based on dominance criteria. Second, it was used to determine whether a female switched her preference after observing the dominance relationship within a dyad of males.

There were two irregularities in the data collection. First, for one CA-like female, the data for the Control 3 could not be collected. A storm during the prior night had blown open the doors to the stimulus subsections, so the female had been exposed prematurely to visual contact with the two stimulus subsections. Second, for another female, on the morning the Control 2 data were to be taken, flooding made the aviary inaccessible. For that female, Control 2 records were taken the following day, as the extra time allowed to acclimate was thought not pivotal.

\section{Data Analysis}

We performed data analysis in a series of nested stages. In stage one, we tested each female for her location preference using a within-bird analysis. We did this in one of two ways, depending on the question of interest; both ways used the large sample approximation (i.e. normal theory approximation) of the two-tailed binomial distribution ( $N=$ observations; Hollander \& Wolfe 1973) with a 5\% criterion level. For the hypothesis testing concerning whether a female had a location bias in the absence of a dyad of males, the comparison frequency for the binomial was 0.5 . For the hypothesis testing involving whether a female had a location bias with a dyad of males present, the comparison frequency was the location frequency of the female from the appropriate control. The changed comparison frequency for the latter set of hypothesis testing most conservatively standardized for any within-bird bias for a side of the 
response chamber. If a female's location preference differed at all from the random expectation of 15 observations on each side (i.e. a location frequency of 0.5 ), we used that deviation in subsequent determinations, regardless of size (i.e. a statistically significant deviation not required).

In stage two, we combined the within-bird results (e.g. preference or no preference) for each of the hypothesis testing situations (i.e. treatments) mentioned above and detailed below. Because this stage included only 'preference' or 'no preference' data for each female (i.e. considered all 'preferences' equally), females with underlying opposite preferences (addressed in stage three analysis) did not 'cancel each other out'. We used the one-tailed binomial distribution ( $N=$ females) for the acrosstreatment (i.e. all of the females under a given treatment) analyses. We then performed three separate analyses for CA-like females, BC-like females, and all females combined.

In stage three, we used subsets of the stage two analyses to address further hypothesis testing. We used the twotailed binomial distribution ( $N=$ females) for these additional across-treatment analyses, except one hypothesis test that had an a priori directionality. Because all of these stage three analyses focused only on females that had previously demonstrated a preference for one male or the other, they all had sample sizes less than 10 and 20, respectively, for each species separately and for the two species combined.

Finally, we performed two tests to assess male dominance interactions. First, we used the two-tailed binomial distribution ( $N=$ dyads) to determine whether dominance within a dyad was related to species. Second, we used the Mann-Whitney $U$ test $\left(N_{1}=\right.$ number of dyads of males with BC-like male dominant, $N_{2}=$ number of dyads of males with CA-like male dominant) to determine whether dominance within a dyad was related to the ratio of male weights.

\section{Ethical Note}

This project was performed under The Ohio State University's ILACUC protocol 97A016. The birds were banded and bled under federal banding permit 20653 and Ohio banding and collecting permit 509, and were held for longer than $24 \mathrm{~h}$ under federal collecting permit MB673317-0.

None of the birds was held captive for longer than a month. The physical interactions within each dyad of males never resulted in physical contact. The most extreme interaction was one sustained chase of approximately $1 \mathrm{~min}$. All birds were released into woodlots at the end of their participation in the experiment, and many were resighted or recaptured during a subsequent experiment.

\section{RESULTS}

We attempted to obtain genetically pure BC and CA individuals. Based on their plumage scores, the large
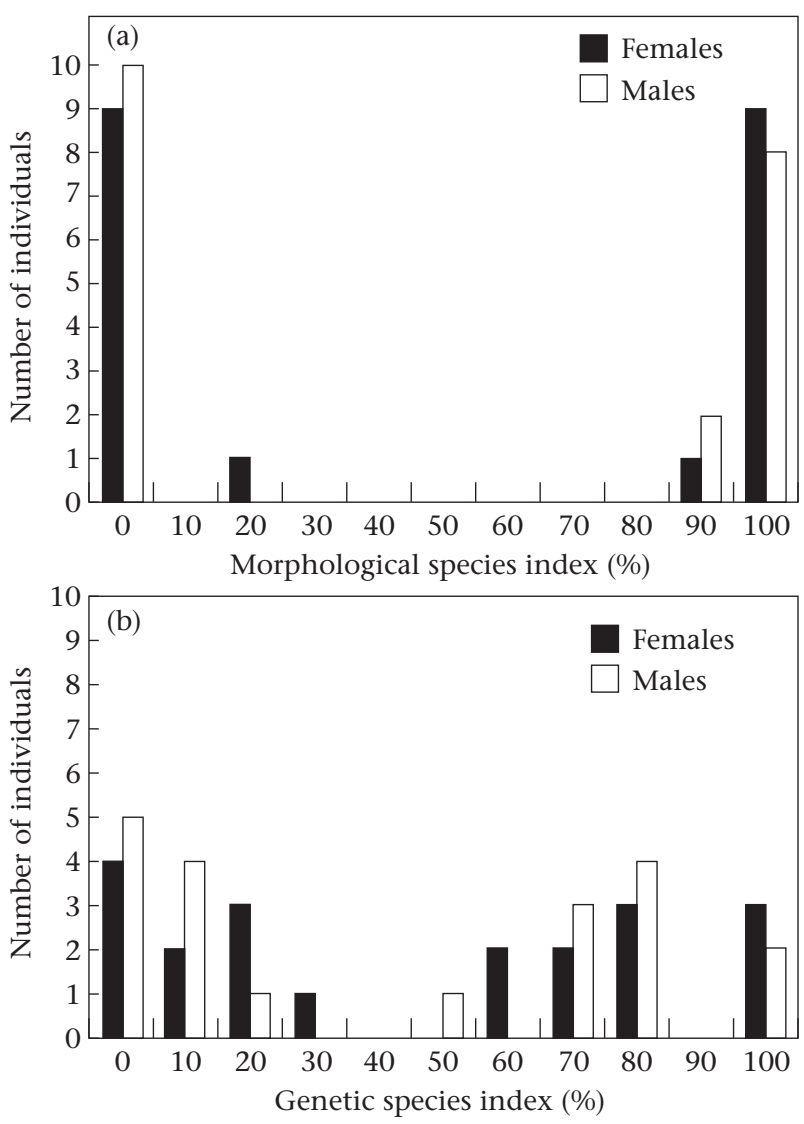

Figure 2. Morphological (a) and genetic indices (b) of male and female chickadees used in a female mate preference experiment. For both indices, pure BC and pure CA would have index values of $0 \%$ and $100 \%$, respectively.

majority (36 of 40) of the birds used had pure BC or CA (Fig. 2a). Only one BC-like individual did not exhibit a pure morphological phenotype, its secondary wing coverts being tan instead of white. Only three of the CA-like individuals did not exhibit a pure morphological phenotype, all had tan secondary feather margins instead of grey.

Contrary to the morphological index, postexperiment genetic analysis indicated that the majority of individuals (26 of 40) showed some level of introgression (Fig. 2b). Of the $\mathrm{BC}$-like individuals, four females and five males had all the BC alleles at the five marker loci ( $0 \%)$, two females and four males had all but one BC variant, three females and one male had all but two BC alleles, and one female had all but four BC alleles. Of the CA-like individuals, three females and two males had all the CA alleles (100\%), three females and four males had all but one CA variant, two females and three males had all but two CA alleles, two females had all but three CA alleles, and one male had all but four CA alleles. Nevertheless, the distribution of the genetic species index was clearly bimodal (Fig. 2b).

Because the observation session occurred approximately $20 \mathrm{~h}$ after the birds were initially exposed to each treatment, only typical flock behaviour was seen in the experimental trio (i.e. contact calls). We employed the 
extended period of acclimation both to eliminate time of day from confounding the differences between components (controls, treatments) and to lower the likelihood of overt interactions still occurring during the observation time.

\section{Female Side Preferences Without Males Present}

\section{Without visual access to the stimulus subsections}

Based on Control 1, we tested individual females (stage one analysis) to determine whether they had a left-right position preference without visual access to the stimulus subsections when no dyad of males was present. The null hypothesis was that a female would not prefer either the left or right side. The alternative hypotheses were that a female would prefer either the left or right side. We combined the results from all the females (no preference or preference) first into the two species categories (10 BC or $10 \mathrm{CA}$ ) and then as one inclusive group (all 20). We tested these pooled data (stage two analysis) to determine whether females (BC, CA or all) had a left-right position preference without visual access to the stimulus subsections when no dyad of males was present. The null hypothesis was that females would not prefer either the left or right side. The alternative hypothesis was that females would prefer a side of the aviary.

The BC-like (binomial test: $P=0.62$ ), CA-like (binomial test: $P=0.17$ ) or the aggregate (binomial test: $P=0.25$ ) of females did not show a left-right position preference when they had no visual access to the stimulus subsections and when a dyad of males was absent. Individually (stage one analysis), three BC-like females significantly preferred the right side of the response section, two significantly preferred the left side, and five had no significant preference. Individually (stage one analysis), four CA-like females significantly preferred the right side of the response section, three significantly preferred the left side, and three had no significant preference.

\section{With visual access to the stimulus subsections}

Based on Control 2, we tested individual females (stage one analysis) to determine whether they had a left-right position preference with visual access to the stimulus subsections when no dyad of males was present. The null hypothesis was that a female would not prefer either the left or right side. The alternative hypotheses were that a female would prefer either the left or right side. We combined the results from all the females (no preference or preference) as before. These pooled data (stage two analysis) were tested to determine whether females had a left-right position preference with visual access to the stimulus subsections when no dyad of males was present. The null hypothesis was that females would not prefer either the left or right side. The alternative hypothesis was that females would prefer a side of the aviary.

The BC-like (binomial test: $P=0.62$ ), CA-like (binomial test: $P=0.17$ ) or the aggregate (binomial test: $P=0.41$ ) of females did not show a left-right position preference when they had visual access to the stimulus subsections and when a dyad of males was absent. Individually (stage one analysis), four BC-like females significantly preferred the right side of the response section, one significantly preferred the left side, and five had no significant preference. Individually (stage one analysis), six CA-like females significantly preferred the right side of the response section, one significantly preferred the left side and three had no significant preference.

\section{Females Preference Based on Nonvisual Male Characteristics}

Based on Control 3 against Control 1, we tested individual females (stage one analysis) to determine whether they preferred one male of the dyad without visual access to the stimulus subsections when a dyad of males was present. The null hypothesis was that a female would not prefer one male of the dyad. The alternative hypotheses were that a female would prefer one male of the dyad. We combined the results from all the females (no preference or preference) first into the two species categories. We tested these pooled data (stage two analysis) to determine whether females preferred one male of the dyad without visual access to the stimulus subsections when a dyad of males was present. The null hypothesis was that females would not prefer one male of the dyad. The alternative hypotheses were that females would prefer one male of the dyad.

BC-like and CA-like females both tended to prefer one or the other male of a dyad based on nonvisual characteristics of the males. BC-like females (nine of 10; binomial test: $P=0.01$ ) significantly preferred one male of a dyad based on nonvisual characteristics. While having a similar tendency, CA-like females (seven of nine; binomial test: $P=0.09$ ) did not show a significant preference for either male of a dyad. When both species were combined, females (16 of 19 ; binomial test: $P<0.01$ ) significantly preferred one male of a dyad based on nonvisual characteristics.

\section{Is there a species-based preference?}

From the pooled data, we tested females that showed a preference (stage three analysis) to determine whether they (either BC, CA or all) had a species-based preference without visual access to the stimulus subsections when a dyad of males was present. The null hypothesis was that females would not prefer either species category. The alternative hypotheses were that females would prefer one of the species categories.

The BC-like (binomial test: $P=0.51$ ), CA-like (binomial test: $P=0.73$ ) or the aggregate (binomial test: $P=0.80$ ) of females that significantly preferred one male of a dyad based on nonvisual characteristics did not show a species preference. Individually (stage one analysis), three BC-like females significantly preferred the BC-like male and six significantly preferred the CA-like male. Individually (stage one analysis) four CA-like females significantly preferred the BC-like male and three significantly preferred the CA-like male. 


\section{Is there a dominance-based preference?}

From the pooled data, we tested females that showed a preference (stage three analysis) to determine whether they had a dominance-based preference without visual access to the stimulus subsections when a dyad of males was present. The null hypothesis was that females would not prefer either dominance type. The alternative hypotheses were that females would prefer one of the dominance types.

The BC-like (binomial test: $P=0.75$ ), CA-like (binomial test: $P=0.07$ ) or the aggregate (binomial test: $P=0.22$ ) of females that significantly preferred one male of a dyad based on nonvisual characteristics did not show a social dominance preference prior to observing the males physically interact. Individually (stage one analysis), five BC-like females significantly preferred the socially dominant male and four significantly preferred the socially subordinate male. Individually (stage one analysis), six CA-like females significantly preferred the socially dominant male and one significantly preferred the socially subordinate male.

\section{Female Preferences Before Observing Male-Male Interactions}

Based on Treatment 1 against Control 2, we tested individual females (stage one analysis) to determine whether they had a preference for one male of the dyad without having observed them physically interact. The null hypothesis was that a female would not prefer either male of the dyad. The alternative hypotheses were that a female would prefer either one of the males. We combined the results from all the females (no preference or preference) first into the two species categories (10 BC or $10 \mathrm{CA}$ ) and then as one inclusive group (all 20). We tested these pooled data (stage two analysis) to determine whether females had a preference for one male of the dyad without having observed them physically interact. The null hypothesis was that females would not prefer either male of the dyad. The alternative hypotheses were that a female would prefer either of the males.

Once in visual contact with the males, both (eight of 10 for each based on stage one analysis) BC-like (binomial test: $P=0.05$ ) and CA-like (binomial test: $P=0.05$ ) females showed a significant preference for one male of a dyad without having observed the males physically interact.

\section{Is there a species-based preference?}

From the pooled data, we separated the females that showed a preference into a group (stage three analysis) and tested them to determine whether they had a speciesbased preference without having observed the dyad of males physically interact. The null hypothesis was that females would not prefer either species category. The alternative hypotheses were that females would prefer one of the species categories.

Neither the BC-like (binomial test: $P=0.29$ ) females nor the CA-like (binomial test: $P=0.07$ ) females that significantly preferred one male of a dyad significantly preferred one species, although six of eight and seven of eight, respectively, did prefer the BC-like male. But females of both species combined (13 of 16 ; binomial test: $P=0.02$ ) significantly preferred the BC-like male without having observed the males physically interact.

\section{Female Preferences After Observing Male-Male Interactions}

\section{Is there a dominance-based preference?}

Based on Treatment 2 against Control 2, we tested individual females (stage one analysis) to determine whether they had a preference for one male of the dyad after having observed them physically interact. The null hypothesis was that a female would not prefer either male of the dyad. The alternative hypotheses were that a female would prefer either one of the males. We combined the results from all the females with a preference (preferring dominant or preferring subordinate) first into the two species categories and then as one inclusive group. We used these pooled data (stage two analysis) to determine whether females had a preference for the socially dominant male of the dyad. The null hypothesis was that females would not prefer the socially dominant male of the dyad. The alternative hypothesis was that females would prefer the socially dominant male of the dyad.

Both BC-like (binomial test: $P=0.04$ ) and CA-like (binomial test: $P=0.04$ ) females showed a significant preference for the socially dominant male of a dyad after observing the males physically interact. In stage one analysis, for both BC-like and CA-like females, eight significantly preferred the dominant male, one significantly preferred the subordinate male, and one had no significant preference.

\section{Do Females Switch Their Preference?}

Based on Treatment 2 against Treatment 1, we tested individual females (stage one analysis) to determine whether they switched their preference for one male of the dyad after having observed them physically interact. The null hypothesis was that a female would not switch her preference for a male of the dyad. The alternative hypotheses were that a female would switch her preference for one of the males. We combined the results from all the females with a preference (switching or not switching) first into the two species categories and then as one inclusive group. We used these pooled data (stage two analysis) to determine whether females switched their preference after observing the males physically interact. The null hypothesis was that females would not switch their preference. The alternative hypothesis was that females would switch their preference after observing the dyad of males physically interact.

None of the female groupings showed a significent tendency to switch their preference after observing the males of a dyad physically interact (binomial test: BC-like: $P=0.50)$, CA-like : $P=0.25$; aggregate: $P=0.24$ ). Individually (stage one analysis), five BC-like females significantly changed their preference for a male, four 
significantly maintained their preference for a male, and one had no significant preference. Individually (stage one analysis), six CA-like females significantly changed their preference for a male, three significantly maintained their preference for a male, and one had no significant preference.

\section{Do females switch their preference to the socially} dominant male?

From the pooled data, we separated the females that switched their preference into a group (stage three analysis) and tested them to determine whether they switched to the socially dominant male after observing the dyad of males physically interact. The null hypothesis was that females would not switch their preference to the socially dominant male. The alternative hypothesis was that females would switch their preference to the socially dominant male.

Of the females that switched their preference after watching the dyad of males physically interact, both BC-like (binomial test: $P=0.03$ ) and CA-like (binomial test: $P=0.11)$ females tended to switch from the subordinate to the dominant male. Individually (stage one analysis), all five BC-like females that switched their preference changed to the dominant male. Individually (stage one analysis), five of the six CA-like females that switched their preference for a male changed to the dominant male. In the aggregate (binomial test: $P=0.01$ ), females of the two species significantly switched their preference to the dominant male after observing the males physically interact.

\section{Do females switch to or maintain their preference for the dominant male?}

Additionally, we used the pooled female data (maintained/switched preference for socially dominant or maintained/switched preference for socially subordinate) from Treatment 2 against Treatment 1, to determine whether females maintained or switched their preference to the dominant male. The null hypothesis was that females would not preferentially maintain or switch their preference based on social dominance. The alternative hypothesis was that females would maintain or switch their preference based on social dominance.

Both BC-like (binomial test: $P=0.02$ ) and CA-like (binomial test: $P=0.10$ ) females tended to maintain or switch their preference to the dominant male of a dyad. The BC-like females and the aggregate (binomial test: $P=0.001$ ) were significant. Individually (stage one analysis), nine of 10 BC-like females maintained or switched their preference to the dominant male, while one female maintained or switched her preference to the subordinate male. Individually (stage one analysis), eight of 10 CA-like females maintained or switched their preference to the dominant male, while two females maintained or switched their preference to the subordinate male. In the aggregate, 17 of 20 females of the two species maintained or switched their preference to the dominant male, while three females maintained or switched their preference to the subordinate male.

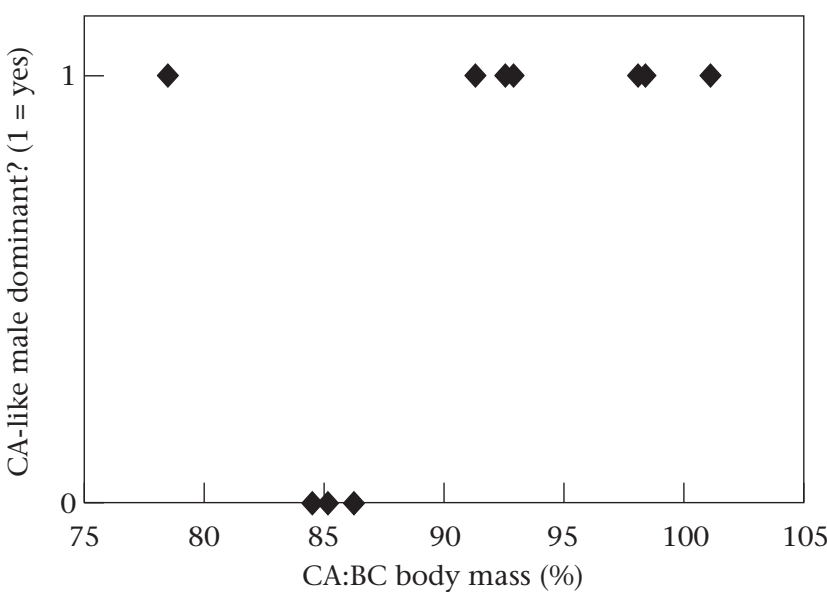

Figure 3. Social dominance of CA-like males over a BC-like males in relation to the ratio of body masses.

\section{Male Dominance Interactions}

Across the two observational periods of physical interaction of the dyad of males, the dominance relationship never changed. The CA-like male was dominant in seven of the 10 dyads (binomial test: $P=0.17$; Fig. 3 ). In the three dyads where the BC-like male was dominant, the CA-like male was noticeably smaller. For the seven pairs in which the CA-like male was socially dominant, the average within-dyad ratio of CA-like to BC-like body mass $(\bar{X} \pm \mathrm{SE}=93.16 \pm 7.44 \%)$ was greater than that for the three dyads in which the BC-like male was socially dominant $(\bar{X} \pm \mathrm{SE}=85.27 \pm 0.86 \%)$ (Fig. 3). The difference between the two ratios closely approached significance (Mann-Whitney $U$ test: $U=3, N_{1}=3, N_{2}=7, P=0.11$ ).

\section{DISCUSSION}

The postexperiment genetic identification of some individuals as impure BC or CA was not unexpected. Sattler \& Braun (2000) found that genetic introgression exceeds morphological introgression in Appalachian transects of this hybrid zone. Also, the northward progression of the Ohio portion of the hybrid zone into the $\mathrm{BC}$ range has potentially left $\mathrm{BC}$ alleles in the wake of the movement. The greater proportion of pure BC individuals (nine of 20) than pure CA individuals (five of 20) could support the hypothesis that the $\mathrm{BC}$ alleles within the $\mathrm{CA}$ individuals are the residual remnants of a previously more southern BC distribution (e.g. Shaw et al. 1993). Additionally, a wide genetic range of individuals exists within the Ohio hybrid zone (C. L. Bronson, unpublished data). This diversity of individuals could indicate widespread interbreeding between the two species and might increase the likelihood of foreign alleles spreading farther from the centre of the hybrid zone. Regardless of the cause, the limited genetic impurity in these experimental individuals would, if anything, bias against observing speciesdriven significant results. The two species samples were not as distinctly different genetically as morphological identification indicated. For a behavioural difference between these two samples to be detected, the 
corresponding difference between two genetically pure samples would have been greater. Therefore, the results presented are conservative.

Sex, size, age and precedence are known to affect the dominance of a dyad of chickadee individuals (Smith 1991). In our protocol, either these factors were not relevant or we attempted to control for them. First, since it was the same within a dyad of males, sex likely did not influence the relationship. Second, our analysis shows that relative size is a contributing factor to the dominance relationship. We tried to use males of similar size. Finally, we contend that precedence links the age and seniority effects. Typically, old birds dominate younger birds, especially in males (Smith 1991); however, older birds have also typically been established longer in a flock, and hence also have seniority. The studies of seniority effects across Paridae tend to involve juveniles and show that in individuals of similar age, the first to arrive ranks higher (black-capped chickadees: Glase 1973; marsh tits, Parus palustris: Nilsson \& Smith 1985, 1988; Nilsson 1989a, b; tufted titmice: Brawn \& Samson 1983; willow tits, P. montanus: Hogstad 1987). Thus, in studies where age was controlled, seniority was the principal factor determining dominance status. This experiment carefully controlled for seniority, and in doing so, we contend, controlled for any potential effects of differences in the ages of the males.

Reproductive history can influence a female's mate choice. For instance, infrequently an older female can remain with her subordinate mate, allowing a younger female to pair with the alpha male (Glase 1973; Ficken et al. 1990; Smith 1991). While we were not able to determine the reproductive history of the experimental females, we believe the protocol limited any potential influence of such history. Since a female had been removed from contact with all previously known males, her mate was effectively dead. She was introduced to a new 'flock'. Because chickadees form pairs in their winter flocks (Ficken et al. 1981; Smith 1991), a female would presumably need to reacquire a mate. Thus, it seems reasonable to assume that, regardless of their previous reproductive history, all of the females in our experiment should have reacted similarly.

The behaviour of females of both chickadee species supported biased mate choice as a potential mechanism for the movement of the Ohio portion of the hybrid zone. In the aggregate, before observing the dominance interaction of the males, females preferred BC-like males. This preference, if it were the sole factor, would tend to cause the hybrid zone to move towards the CA range, opposite to the observed direction. Unfortunately, the design of this experiment did not allow for specifically attributing the preference to any BC-like morphological phenotype independent of body size.

In the aggregate, both BC-like and CA-like females preferred the dominant male to the subordinate. Females could gain several benefits from associating with a more dominant mate. First, a high-dominance pair has a better chance of obtaining a larger (Smith 1976) and/or higherquality (Smith 1991; Otter et al. 1999) breeding territory. Second, the dominance status of a female's mate influences her winter survival more than does her own dominance level (Ekman 1990; Hogstad 1992; Lemmon et al. 1997).

Aside from obtaining a high-dominance mate, female preference for dominant males also influences choice of extrapair mating partners (Otter et al. 1998). BC females actively seek males of higher rank than their own mate for extrapair copulations (EPCs) (Smith 1988). They also choose nest sites close to territories of high-ranking males, thus, increasing their chances of an EPC with a high-ranking male (Ramsay et al. 1999).

Based on the relatively quick identification of dominance by a human observer (under 30 min for all dyads), we were not able to develop a measure for potential differences in the relative dominance between different dyads. For instance, one particular heterospecific dyad might have been between sequentially ranked males if placed within a larger group of males, whereas another heterospecific dyad might have had a few individuals between them in a larger group. Based on the short time frame it took to categorize the dominant male, we assume that all the dyads were of relatively equal asymmetry. However, if females do have a requisite threshold for dominance to be important, our results are conservative. For example, in closely 'ranked' dyads, females may not change their preference to the dominant male from the subordinate. Within this experiment, such females would be categorized as 'not switching to the dominant male' and the likelihood of detecting a significant relationship between dominance and switching would be decreased.

The dominance observed in the aviary is likely to also exist in a natural setting since aviary dominance interactions in $\mathrm{BC}$ have been shown to be behaviourally similar to those at feeders (Lempriere 1990). Additionally, dominance hierarchies observed at feeders (two present in the stimulus section of the aviary) and away from them in nature are identical (Smith 1976).

In nature, both species form within-sex dominance hierarchies. While studies in BC have not shown a relationship between weight and rank (Glase 1973; Smith 1976), neither of the studies controlled for known variation in weight (e.g. season, time of day). In willow tits, when daily weight fluctuations were limited, weight explained $77 \%$ of variance in rank (Hogstad 1987). In the aviary, the tendency for CA-like males to dominate BC-like males of similar size could lead to a preference in females of both species for CA-like males. Across a northsouth transect within the distribution of either parental species, both species display a positive relationship between body size and latitude (Lunk 1952; Brewer 1961). Within areas where their distributions abut, these size clines tend to overlap and the species are generally of similar size (Lunk 1952; Brewer 1961). Assuming that any CA-like male likely to be encountered by a BC-like female in the hybrid zone would be about as large as a BC-like male, the observed preference for the dominant male would cause a movement of the hybrid zone in a northward direction, as observed.

Female preferences for BC-like males, on the one hand, and dominant males, on the other hand, could potentially negate each other. The design of the experiment 
allowed the relative strengths of these two preferences to be assessed. Based on existing knowledge of conspecific mate choice in chickadees, social dominance was assumed a priori as the more important factor and was presented second. The females did maintain or switch from their initial preference to the dominant male, indicating that within-sex dominance status was more influential than any species-specific male attributes.

Either of two mechanisms could have been responsible for females maintaining or switching their preference to the dominant male after observing the two males interact. Females of both species might have preferred the BC morphological phenotype in the absence of other cues. Alternatively, in the absence of directly observing males interact, the females might have used a surrogate cue (e.g. body size) as an indicator of dominance. In nine of 10 male dyads, the $\mathrm{BC}$ male was larger than the CA male.

Near and within the hybrid zone, the two species and their hybrids are of similar size (Lunk 1952; Brewer 1961) and the two species show interspecific territorial defence (Brewer 1961). The combination of female preference for dominant individuals (Smith 1991; Otter \& Ratcliffe 1996; Ramsay et al. 2000) and dominant individuals commanding better territories (Smith 1991; Otter et al. 1999) would appear to promote movement of the zone in the observed direction, towards the $\mathrm{BC}$ range. A similar process of male aggression affecting the movement of an avian hybrid zone was indicated in an experimental study of white-collared manakins, Manacus candei, and goldencollared manakins, M. vitellinus (McDonald et al. 2001). The greater aggression of the golden-collared manakins has been suggested as the cause for introgression of golden-collared plumage traits into the range of the white-collared manakin (McDonald et al. 2001).

While potentially providing some insight into the northward movement of the hybrid zone in Ohio, this experiment suggests several avenues for further investigation. For example, what criteria are the females using in the absence of visual information to make a choice? Both species tended to show a preference when visually isolated from the males. The preference was not associated with the species or dominance of the males. Some other factor needs to be identified. Also, do females really prefer the $\mathrm{BC}$ morphological phenotype independent of body size? Possibly, CA males altered to the $\mathrm{BC}$ morphological phenotype would be preferred over the CA morphological phenotype. Also, in hybrids, is the CA tendency for male dominance linked to the CA morphological phenotype, or could 'superhybrids' exist with the BC morphological phenotype, but the CA capacity to dominate social interactions?

\section{Acknowledgments}

Thanks to Andrew Dolby, Tim Kloth and Elena Pravosudova for assistance in obtaining the birds, to Nidia Arguedas, Robb Brumfield and Chris Huddleston for assistance in the laboratory, and to Sandra Gaunt, Douglas Nelson, Patricia Parker, the OSU Behavioral
Ecology Group and the SI-LMS lunch group for discussion and comments. This study was funded by NSF grant IBN-9522064 to T.C.G.

\section{References}

Arntzen, J. W. \& Wallis, G. P. 1991. Restricted gene flow in a moving hybrid zone of the newts Triturus cristatus and T. marmoratus in western France. Evolution, 45, 805-826.

Bert, T. M. \& Harrison, R. G. 1988. Hybridization in western Atlantic stone crabs (genus Menippe): evolutionary history and historical context influence species interactions. Evolution, 42, 528-544.

Braun, M. J. \& Robbins, M. B. 1986. Extensive protein similarity of the hybridizing chickadees Parus atricapillus and P. carolinensis. Auk, 103, 667-675.

Brawn, J. D. \& Samson, F. B. 1983. Winter behavior of tufted titmice. Wilson Bulletin, 95, 222-232.

Brewer, R. 1961. Comparative notes on the life history of the Carolina chickadee. Wilson Bulletin, 73, 348-373.

Burley, N. 1986. Sexual selection for aesthetic traits in species with biparental care. American Naturalist, 127, 415-445.

Burley, N. 1988. Wild zebra finches have band-colour preferences. Animal Behaviour, 36, 1235-1237.

Desrochers, A. 1990. Sex determination of black-capped chickadees with a discriminant analysis. Journal of Field Ornithology, 61, 79-84.

Dowling, T. E., Moritz, C. \& Palmer, J. D. 1990. Nucleic acids II: restriction site analysis. In: Molecular Systematics (Ed. by D. M. Hillis \& C. Moritz), pp. 250-317. Sunderland, Massachusetts: Sinauer.

Ekman, J. 1990. Alliances in winter flocks of willow tits: effects of rank on survival and reproductive success in male-female association. Behavioral Ecology and Sociobiology, 26, 239-245.

Ficken, M. S., Witkin, S. R. \& Weise, C. M. 1981. Associations among members of a black-capped chickadee flock. Behavioral Ecology and Sociobiology, 8, 245-249.

Ficken, M. S., Weise, C. M. \& Popp, J. W. 1990. Dominance and resource access in winter flocks of black-capped chickadees. Wilson Bulletin, 102, 623-633.

Glase, J. C. 1973. Ecology of social organization in the black-capped chickadee. Living Bird, 12, 235-267.

Grubb, T. C., Jr \& Bronson, C. L. 1995. Artificial snags as nesting sites for chickadees. Condor, 97, 1067-1070.

Grubb, T. C., Jr, Mauck, R. A. \& Earnst, S. L. 1994. On no-chickadee zones in Midwestern North America: evidence from the Ohio Breeding Bird Atlas and the North American Breeding Bird Survey. Auk, 111, 191-197.

Hairston, N. G., Sr, Wiley, R. H., Smith, C. K. \& Kneidel, K. A. 1992. The dynamics of two hybrid zones in Appalachian salamanders of the genus Plethodon. Evolution, 46, 930-938.

Harrison, R. G. 1990. Hybrid zones: windows on evolutionary process. Oxford Survey of Evolutionary Biology, 7, 69-128.

Harrison, R. G. 1993. Hybrid Zones and the Evolutionary Process. New York: Oxford University Press.

Hartzler, J. E. 1970. Winter dominance relationship in black-capped chickadees. Wilson Bulletin, 82, 427-434.

Hewitt, G. M. 1988. Hybrid zones: natural laboratories for evolutionary studies. Trends in Ecology and Evolution, 3, 158-167.

Hollander, M. \& Wolfe, D. A. 1973. Nonparametric Statistical Methods. New York: J. Wiley.

Hogstad, O. 1987. Social rank in winter flocks of willow tits, Parus montanus. Ibis, 129, 1-9.

Hogstad, O. 1992. Mate protection in alpha pairs of wintering willow tits, Parus montanus. Animal Behaviour, 43, 323-328. 
Johnson, K., Dalton, R. \& Burley, N. 1993. Preferences of female American goldfinches (Carduelis tristis) for natural and artificial traits. Behavioral Ecology, 4, 138-143.

Kallioinen, R. U. O., Hughes, J. M. \& Mather, P. B. 1995. Significance of black colour in territorial interaction in Australian magpies. Australian Journal of Zoology, 44, 665-673.

Lehner, P. N. 1979. Handbook of Ethological Methods. New York: Garland STPM Press.

Lemmon, D., Withiam, M. L. \& Barkan, C. P. 1997. Mate protection and winter pair bonds in black-capped chickadees. Condor, 99, 424-433.

Lempriere, C. S. 1990. Plasticity in the chickadee call of wintering flocks of black-capped chickadees (Parus atricapillus). MSc. thesis, Queen's University, Kingston, Canada.

Li, Y., Turck, C. M., Teumer, J. K. \& Stavnezer, E. 1986. Unique sequence, ski, in Sloan-Kettering avian retrovirus with properties of a new cell-derived oncogene. Journal of Virology, 57, 1065-1072.

Longmire, J. L., Lewis, A. K., Brown, N. C., Buckingham, J. M., Clark, L. M., Jones, M. D., Meincke, L. J., Meyne, J., Ratliff, R. L., Ray, F. A., Wagner, R. P. \& Moyzis, R. K. 1988. Isolation and molecular characterization of a highly polymorphic centromeric tandem repeat in the family Falconidae. Genomics, 2, 14-24.

Lunk, W. A. 1952. Notes on variation in the Carolina chickadee. Wilson Bulletin, 64, 7-21.

McDonald, D. B., Clay, R. P., Brumfield, R. T. \& Braun, M. J. 2001 Sexual selection on plumage and behavior in an avian hybrid zone: experimental tests of male-male interactions. Evolution, 55, 1443-1451.

Mack, A. L., Gill, F. B., Colburn, R. \& Spolsky, C. 1986. Mitochondrial DNA: a source of genetic markers for studies of similar passerine bird species. Auk, 103, 676-681.

Metz, K. J. \& Weatherhead, P. J. 1991. Color bands function as secondary sexual traits in male red-winged blackbirds. Behavioral Ecology and Sociobiology, 28, 23-27.

Nilsson, J.-Å. 1989a. Causes and consequences of natal dispersal in the marsh tit, Parus palustris. Journal of Animal Ecology, 58, 619-636.

Nilsson, J.-Å. 1989b. Establishment of juvenile marsh tits in winter flocks: an experimental study. Animal Behaviour, 38, 586-595.

Nilsson, J.-Å. \& Smith, H. G. 1985. Early fledging mortality and the timing of juvenile dispersal in the marsh tit, Parus palustris. Ornis Scandinavica, 16, 293-298.

Nilsson, J.-Å. \& Smith, H. G. 1988. Effects of dispersal date on winter flock establishment and social dominance in marsh tits, Parus palustris. Journal of Animal Ecology, 57, 917-928.

Otter, K. A. \& Ratcliffe, L. M. 1996. Female initiated divorce in a monogamous songbird: abandoning mates for males of higher quality. Proceedings of the Royal Society of London, Series B, 263, 351-355

Otter, K., Ratcliffe, L., Michaud, D. \& Boag, P. T. 1998. Do female black-capped chickadees prefer high-ranking males as extra-pair partners? Behavioral Ecology and Sociobiology, 43, 25-36.

Otter, K. A., Ramsay, S. M. \& Ratcliffe, L. M. 1999. Enhanced reproductive success of female black-capped chickadees mated to high-ranking males. Auk, 116, 345-354.
Pearson, S. F. 2000. Behavioral asymmetries in a moving hybrid zone. Behavioral Ecology, 11, 84-92.

Pearson, S. F. \& Manuwal, D. A. 2000. Influence of niche overlap and territoriality on hybridization between hermit and Townsend's warblers. Auk, 117, 175-183.

Pearson, S. F. \& Rohwer, S. 2000. Asymmetries in male aggression across an avian hybrid zone. Behavioral Ecology, 11, 95-101.

Peterjohn, B. G. 2001. The Birds of Ohio. Wooster, Ohio: Wooster.

Pierce, V. \& Grubb, T. C., Jr. 1979. Laboratory studies of foraging in four bird species of deciduous woodland. Auk, 98, 307-320.

Pyle, P. 1997. Identification Guide to North American Birds. Bolinas, California: Slate Creek Press.

Ramsay, S. M., Otter, K. A. \& Ratcliffe, L. M. 1999. Nest-site selection by female black-capped chickadees: settlement based on conspecific attraction? Auk, 116, 604-617.

Ramsay, S. M., Otter, K. A., Mennill, D. J., Ratcliffe, L. M. \& Boag, P. T. 2000. Divorce and extra pair mating in female black-capped chickadees (Parus atricapillus): separate strategies with a common target. Behavioral Ecology and Sociobiology, 49, 18-23.

Rohwer, S. \& Wood, C. 1998. Three hybrid zones between hermit and Townsend's warblers in Washington and Oregon. Auk, 115, 284-310.

Sambrook, J., Fritsch, E. F. \& Maniatis, T. 1989. Molecular Cloning: a Laboratory Manual. 2nd edn. Cold Spring Harbor, New York: Cold Spring Harbor Laboratory Press.

Sattler, G. D. 1996. The dynamics of vocal, morphological, and molecular interaction between hybridizing black-capped and Carolina chickadees. Ph.D. thesis, University of Maryland.

Sattler, G. D. \& Braun, M. J. 2000. Morphometric variation as an indicator of genetic interactions between black-capped and Carolina chickadees at a contact zone in the Appalachian Mountains. Auk, 117, 427-444.

Sawaya, P. L. 1990. A detailed analysis of the genetic interaction at a hybrid zone between the chickadees Parus atricapillus and $P$. carolinensis as revealed by nuclear and mitochondrial DNA restriction fragment length variation. Ph.D. thesis, University of Cincinnati.

Shapiro, L. H. 1998. Hybridization and geographic variation in two meadow katydid contact zones. Evolution, 52, 784-796.

Shaw, D. D., Marchant, A. D., Contreras, N., Arnold, M. L., Groeters, F. \& Kohlmann, B. C. 1993. Genomic and environmental determinants of a narrow hybrid zone: cause or coincidence? In: Hybrid Zones and the Evolutionary Process (Ed. by R. G. Harrison), pp. 165-195. New York: Oxford University Press.

Smith, S. M. 1976. Ecological aspects of dominance hierarchies in black-capped chickadees. Auk, 93, 95-107.

Smith, S. M. 1988. Extra-pair copulations in black-capped chickadees: the role of the female. Behaviour, 107, 15-23.

Smith, S. M. 1991. The Black-capped Chickadee: Behavioral Ecology and Natural History. New York: Comstock.

Trautman, M. B. 1940. The birds of Buckeye Lake, Ohio. University of Michigan Museum Zoological Miscellaneous Publication, 44, 310-312.

Wheaton, J. M. 1882. Report on the birds of Ohio. Ohio Geological Survey Bulletin, 4, 187-623. 